\title{
Avaliação da síndrome metabólica em agentes comunitários de saúde em município do norte de Minas Gerais
}

\section{Evaluation of metabolic syndrome in community health agents in a northern Minas Gerais municipality \\ Evaluación del síndrome metabólico de los trabajadores de salud comunitários em el município norteño de Minas Gerais}

Lucinéia de Pinho $^{1 \oplus}$, Rayane Ruas Oliveira ${ }^{\oplus}$, Luciane Gonçalves Pereira ${ }^{1}$, Matheus Oliveira Nobre de Andrade ${ }^{1}$, Ana Natália Oliveira Teixeira ${ }^{1}$, Mariano Fagundes Neto Soares ${ }^{1}$

${ }^{1}$ Centro Universitário FIPMoc (UNIFIPMoc) Ibituruna, Montes Claros - MG

\section{Resumo}

\begin{abstract}
Introdução: A síndrome metabólica se relaciona a doenças crônicas, possui prevalência crescente no Brasil e leva riscos cardiovasculares à população. Objetivo: Comparar a prevalência da síndrome metabólica em agentes comunitários de saúde em município do Norte de Minas Gerais segundo diferentes critérios diagnósticos. Métodos: Foi realizada coleta de dados de 675 profissionais que compunham as variáveis dos critérios diagnósticos propostos pela Internacional Diabetes Federation e National Cholesterol Education Program's Adult Treatment Panel III. Resultados: Segundo critérios da Internacional Diabetes Federation, diagnosticou-se $42,2 \%$ de indivíduos com síndrome metabólica e 33,6\% segundo National Cholesterol Education Program's Adult Treatment Panel III. Há aumento diretamente proporcional ao avançar da idade ( $\mathrm{p}=0,000$ para ambos os critérios) e relação intrínseca à dislipidemia e pressão arterial elevada. Conclusão: Pôde-se concluir que a síndrome metabólica tem prevalência muito relevante no público estudado. Em relação às variáveis analisadas, encontrou-se similaridade entre critérios. Assim, nota-se que essas informações são importantes para realização de um diagnóstico precoce e manutenção da saúde dos agentes comunitários de saúde.
\end{abstract}

Palavras-chave: Dislipidemia; Obesidade Abdominal; Hipertensão; Doenças Cardiovasculares; Resistência à Insulina.

Como citar: Pinho L, Oliveira RR, Pereira LG, Andrade MON, Teixeira ANO, Soares MFN.Avaliação da síndrome metabólica em agentes comunitários de saúde em município do norte de Minas Gerais. Rev Bras Med Fam Comunidade. 2020;15(42):2605. https://doi.org/10.5712/rbmfc15(42)2605
Autor correspondente: Lucinéia de Pinho. E-mail: lucineiapinho@ hotmail.com Fonte de financiamento: declaram não haver.

Parecer CEP:

2.425.756/2017

Procedência: não encomendado. Recebido em: 30/06/2020. Aprovado em: 10/11/2020. 


\begin{abstract}
Introduction: Metabolic syndrome is related to chronic diseases, has an increasing prevalence in Brazil and takes cardiovascular risks to the population. Objectives: To compare the prevalence of metabolic syndrome in community health workers in a municipality in the North of Minas Gerais according to the different diagnostic criteria. Methods: Data were collected from 675 professionals who comprised the variables of the diagnostic criteria proposed by the International Diabetes Federation and National Cholesterol Education Program's Adult Treatment Pane III. Results: According to the criteria of the International Diabetes Federation, $42.2 \%$ of individuals with Metabolic Syndrome and $33.6 \%$ were diagnosed according to the National Cholesterol Education Program's Adult Treatment Panel III. There is a directly proportional increase with advancing age ( $p=0.000$ for both criteria) and an intrinsic relationship to dyslipidemia and high blood pressure. Conclusion: It was concluded that the metabolic syndrome has a very relevant prevalence in the studied public. Regarding the analyzed variables, similarity was found between criteria. Thus, it is noted that this information is important for making an early diagnosis and maintaining the health of community health workers.

Keywords: Dyslipidemia; Abdominal Obesity; Hypertension; Cardiovascular Diseases; Insulin Resistance
\end{abstract}

\title{
Resumen
}

Introducción: El Síndrome Metabólico está relacionado con enfermedades crónicas, tiene una prevalencia creciente em Brasil y conlleva riesgos cardiovasculares para la población. Objetivo: Comparar la prevalencia de síndrome metabólico en trabajadores comunitarios de salud de un municipio del norte de Minas Gerais según los diferentes criterios diagnósticos. Métodos: Se recolectaron datos de 675 profesionales que comprendieron las variables de los criterios de diagnóstico propuestos por la Federación International de Diabetes y el Panel III de Tratamiento de Adultos del Programa Nacional de Educación sobre el Colesterol. Resultados: Según los criterios de la Federación Internacional de Diabetes, el 42,2\% de las personas con Síndrome Metabólico y el 33,6\% fueron diagnosticados de acuerdo con el Panel III de Tratamiento de Adultos del Programa Nacional de Educación sobre el Colesterol. Hay un aumento directamente proporcional con la edad avanzada ( $p=0,000$ para ambos criterios) y una relación intrínseca con la dislipidemia y la presión arterial alta. Conclusión: Se concluyó que el Síndrome Metabólico tiene una prevalencia muy relevante em el público estudiado. En cuanto a las variables analizadas, se encontró similitud entre los criterios. Por lo tanto, se observa que esta información es importante para hacer un diagnóstico temprano y mantener la salud de los agentes de salud de la comunidad.

Palabras clave: Dislipidemia; Obesidad abdominal; Hipertensión; Enfermedades cardiovasculares; Resistencia a la insulina.

\section{INTRODUÇÃO}

A síndrome metabólica (SM) é considerada um importante problema de saúde pública no mundo e no Brasil, devido à sua alta e crescente prevalência, bem como a sua influência na predição de doenças cardiovasculares (DCV), invalidez e morte. Estima-se que a prevalência da SM em todo o mundo se encontra em torno de $25 \%$ na população adulta. ${ }^{1}$ No Brasil, a condição apresenta-se entre 8,4\% a 9,5\%, entretanto, as grandes extensões territoriais, com suas diferentes variações demográficas, epidemiológicas e culturais dificultam a estimativa dos valores em cada região. ${ }^{2}$

A SM é um distúrbio que eleva em cinco vezes o risco de diabetes mellitus tipo 2 e em três vezes o de DCV, bem como predispõe doenças coronarianas e microvasculares. Apesar da existência de análises sobre as doenças crônicas metabólicas e cardiovasculares, tanto a prevalência quanto as características epidemiológicas da síndrome são pouco conhecidas, devido aos diferentes componentes individuais possíveis, a depender do critério diagnóstico a ser utilizado. ${ }^{3}$

A ausência de um consenso entre os diferentes critérios de definição e os pontos de corte de cada um de seus itens levam a diferentes estimativas da prevalência da SM, dificultando também o seu diagnóstico na prática clínica. Estabelecidos pela International Diabetes Federation (IDF), Organização Mundial de Saúde (OMS), American Association of Clinical Endocrinologists (AACE) e pelo National Cholesterol Education Program's Adult Treatment Panel III (NCEP-ATP III), esses são os critérios diagnósticos mais amplamente difundidos para a classificação da SM. ${ }^{1,3,4}$ 
O critério proposto pela IDF tem como objetivo a criação de uma ferramenta universalmente aceita e de fácil aplicação, sem a necessidade de medições mais elaboradas, assim como o do NCEP-ATP III, justificando a importância dessas duas entidades no diagnóstico da SM. Ambos os critérios relacionam suas variáveis à fisiopatologia da síndrome e consideram os principais parâmetros alterados na presença da SM para o seu diagnóstico, como obesidade central, lipoproteína de alta densidade (HDL) reduzida, triglicérides, pressão arterial e glicemia de jejum elevados. A IDF possui algumas particularidades, considerando a obesidade central mais fortemente relacionada com a resistência à insulina do que os outros critérios. . $^{5,6}$

A carência de estudos em trabalhadores da área da saúde, associada à gravidade das comorbidades secundárias à SM na população adulta evidenciam a necessidade da realização de um rastreamento da doença na atenção primária à saúde (APS). ${ }^{7}$ Esse resultado poderá contribuir para intervenções custo efetivas e terapêuticas futuras em saúde pública, que tenham como propósito o diagnóstico precoce, a redução da morbimortalidade e da sobrecarga dos serviços de saúde pelas complicações da síndrome..$^{2,8}$ O objetivo geral do estudo é comparar a prevalência da síndrome metabólica em agentes comunitários de saúde em município do Norte de Minas Gerais segundo os diferentes critérios diagnósticos.

\section{MÉTODOS}

Trata-se de uma pesquisa analítica, transversal e quantitativa, que foi realizada em uma cidade localizada no norte do estado de Minas Gerais. A população foi composta por agentes comunitários de saúde (ACS) atuantes nas estratégias de saúde da família (ESF) da APS com cobertura completa da cidade. No momento da coleta de dados, o referido município possuía 135 ESFs, contemplando 797 agentes comunitários de saúde. Para este estudo estimou-se o tamanho amostral mínimo de 299 ACS, com base no quantitativo de profissionais ACS vinculados ao município, a prevalência do desfecho "síndrome metabólica" igual a 50,0\%, o erro amostral de 5\%, o nível de confiança de $95 \%$ e acréscimo de $15 \%$ para eventuais perdas. Contudo, foram convidados para participar do estudo todos os profissionais do município. O critério de inclusão foi ter pelo menos seis meses de efetivo exercício na função de agente comunitário de saúde no referido município. Foram excluídos os ACS em desvio de função ou de licença por qualquer natureza e na condição de gestante.

A coleta de dados foi realizada durante agosto a outubro de 2018, por entrevistadores previamente capacitados. Inicialmente, os profissionais foram identificados em seus setores de trabalho e convidados a participar do estudo da avaliação da síndrome metabólica em agentes comunitários de saúde. Os ACS foram avaliados nas instalações do centro de referência regional em saúde do trabalhador (CEREST) do município, em dias úteis da semana e no período matutino.

Todos os ACS que optaram por participar do estudo contribuíram voluntariamente para as atividades solicitadas. Nenhum ACS participou do planejamento, desenho e condução do estudo. Os profissionais do CEREST do município contribuíram publicando sobre a importância e os possíveis benefícios do projeto para os ACS. Como forma de retribuição, os autores do presente estudo forneceram os principais resultados e conclusões sobre os dados coletados para o CEREST e para o ACS os resultados dos exames bioquímicos, a fim de fortalecer a política local de saúde do trabalhador.

Para a coleta de dados, utilizou-se um questionário autoaplicável que contemplava as condições sociodemográficas (sexo e idade) e avaliações físicas dos profissionais (medida antropométrica, pressão 
arterial e perfil bioquímico). No quesito idade, a população consultada foi dividida em três grupos, sendo eles: ACS de até 29 anos, de 30 a 39 anos e com idade superior a 39 anos.

Para a avaliação antropométrica foi calculada a circunferência abdominal (CA) através de fita métrica, com variação de $0,1 \mathrm{~cm}$. Levou-se em consideração o ponto médio entre a altura da última costela e a porção superior da crista ilíaca, com o adulto posicionado em ortostatismo e ereto. ${ }^{9}$

A aferição da pressão arterial baseou-se nas recomendações da Sociedade Brasileira de Cardiologia, ${ }^{10}$ através de esfigmomanômetros e estetoscópios, sendo selecionado o manguito de tamanho adequado para a circunferência do braço, seguido pela determinação da pressão sistólica através da ausculta do primeiro som (fase I de Korotkoff) e da pressão diastólica no desaparecimento dos sons (fase $V$ de Korotkoff). Para uma aferição adequada, foi orientado aos pacientes para que descansassem e não falassem por 5-10 minutos, permanecessem em ambiente calmo, com temperatura agradável e com as pernas descruzadas. Foi certificado de que o paciente não estava com a bexiga cheia e que não tinha praticado exercícios físicos, feito uso de bebidas alcoólicas, café ou cigarro por pelo menos 60 minutos antes da aferição.

Os parâmetros metabólicos avaliados foram HDL, triglicérides e glicemia de jejum. Os dados foram obtidos através da coleta de sangue venoso periférico após jejum de oito a doze horas. Os índices de HDL e triglicérides foram analisados através do método colorimétrico enzimático e glicemia pelo método enzimático.

Este estudo contemplou variáveis relativas aos critérios diagnósticos da SM propostos pela IDF e NCEP-ATP III. De acordo com a classificação proposta pela IDF, em 2005, é necessário a presença de obesidade central, sendo avaliada pela circunferência abdominal aumentada: $\geq 90 \mathrm{~cm}$ para homens e $\geq 80 \mathrm{~cm}$ para mulheres da América do Sul, associada a dois ou mais critérios: triglicérides $\geq 150 \mathrm{mg} / \mathrm{dl}$ e/ ou $\mathrm{HDL}<40 \mathrm{mg} / \mathrm{dl}$ nos homens e $<50 \mathrm{mg} / \mathrm{dl}$ nas mulheres ou em tratamento específico; pressão arterial $\geq 130 / 85 \mathrm{mmHg}$ ou em tratamento anti-hipertensivo e glicose $\geq 100 \mathrm{mg} / \mathrm{dl}$ ou presença de diabetes mellitus previamente diagnosticada. ${ }^{5}$

Os critérios do NCEP-ATP III levam em consideração a presença de três de cinco componentes: circunferência abdominal: $>102 \mathrm{~cm}$ em homens e $>88 \mathrm{~cm}$ em mulheres; triglicérides $\geq 150 \mathrm{mg} / \mathrm{dl}$ e/ou $\mathrm{HDL}<40 \mathrm{mg} / \mathrm{dl}$ em homens e $<50 \mathrm{mg} / \mathrm{dl}$ em mulheres; pressão arterial $\geq 130 / 85$ e glicose sérica $\geq 110 \mathrm{mg} / \mathrm{dl} .{ }^{6}$

Os dados foram compilados em planilha eletrônica e, posteriormente codificados e analisados no software Statistical Package For Social Science versão 25.0 (SPSS). Primeiramente, realizou-se uma análise exploratória por meio de estatísticas descritivas, sendo utilizado, para as variáveis categóricas, as frequências absolutas e relativas e, para as variáveis contínuas, o cálculo da média e desvio padrão. Posteriormente, foram identificadas as prevalências da síndrome metabólica pelos métodos IDF e NCEPATP III. A concordância entre os métodos foi verificada pelo índice Kappa e intervalo de confiança (IC 95\%), sendo que os valores abaixo de zero indicam ausência de concordância, entre 0 e 0,20 indicam concordância pobre, entre 0,21 e 0,40 leve concordância, entre 0,41 e 0,60 denotam concordância moderada, 0,61 e 0,80 é considerável e valores entre 0,81 e 1,00 indicam uma concordância quase ideal. ${ }^{11}$

Em seguida, por intermédio de gráficos de frequência relativa, comparou-se a prevalência da síndrome metabólica pelos dois métodos agrupados pela variável sexo e idade. Por fim, dentre os ACS que manifestaram a presença de cada variável que forma os indicadores de SM em ambos os métodos, foi comparada a prevalência de cada componente da síndrome, tanto para o sexo masculino, quanto para o sexo feminino. Para isto, foi utilizado o teste qui-quadrado de Pearson e a significância foi considerada para $p<0,05$. 
Esse estudo foi realizado conforme a Resolução $n^{\circ}$ 510/2016, que dispõe sobre as normas para a realização de pesquisas cuja metodologia envolva diretamente os dados obtidos com os participantes ou informações identificáveis. ${ }^{12} O$ projeto base foi aprovado pelo Comitê de Ética em Pesquisa com Seres Humanos da Universidade Estadual de Montes Claros - Unimontes, com parecer de aprovação $n^{\circ} 2.425 .756 / 2017$ e foi aplicado o termo de consentimento livre e esclarecido e o termo de concordância da instituição para autorização de pesquisa. O banco de dados anonimizado estará disponível para pesquisadores com projeto de pesquisa aprovado em comitê de ética em pesquisa, mediante contato com a autora correspondente.

\section{RESULTADOS}

Do total de 675 agentes analisados no período do estudo, todos participaram da pesquisa, com a faixa etária variando de 19 a 68 anos, sendo 565 (83,70\%) do sexo feminino. As medidas descritivas da circunferência abdominal, parâmetros laboratoriais, pressão arterial e idade foram apresentadas na Tabela 1.

A prevalência da SM no estudo foi de $42,2 \%$ dos agentes comunitários segundo a IDF ( $n=285)$ e de $33,6 \%$ segundo o NCEP-ATP III ( $n=227)$. Observou-se que 32,7\% $(n=221)$ dos participantes apresentaram a SM tanto pelo critério IDF como pelo NCEP-ATP III, e 56,9\% ( $n=384)$ não fecharam o diagnóstico por nenhum dos dois critérios. Essa concordância pode ser confirmada pelo índice Kappa =0,781 [0,734; 0,828] (Tabela 2).

Os critérios IDF $(p=0,467)$ e NCEP-ATP III $(p=0,378)$ mostraram que não diferiram estatisticamente entre o sexo dos indivíduos portadores de SM (Figura 1).

Os critérios IDF $(p=0,000)$ e NCEP-ATP III $(p=0,000)$ mostraram-se estatisticamente significativos em relação à faixa etária da população estudada. Ao comparar os resultados, percebeu-se maior prevalência da SM diretamente proporcional ao aumento da idade dos ACS nos dois critérios avaliados (Figura 2).

Tabela 1. Parâmetros clínicos dos 675 agentes comunitários avaliados por gênero, em uma cidade localizada no norte de Minas Gerais.

\begin{tabular}{lcc}
\hline & Homens $\mathbf{( n = 1 1 0 )}$ & Mulheres $(\mathbf{n}=\mathbf{5 6 5})$ \\
\hline Idade (anos) & $32,94 \pm 10,29$ & $37,44 \pm 9,61$ \\
CA (cm) & $92,61 \pm 14,74$ & $90,16 \pm 13,04$ \\
GJ (mg/dL) & $87,78 \pm 16,31$ & $86,91 \pm 29,57$ \\
PAS (mmHg) & $148,89 \pm 17,03$ & $137,83 \pm 18,61$ \\
PAD (mmHg) & $79,21 \pm 9,86$ & $77,54 \pm 10,69$ \\
TG (mg/dL) & $183,04 \pm 141,81$ & $138,77 \pm 74,01$ \\
HDL-c (mg/dL) & $46,70 \pm 6,73$ & $49,98 \pm 8,41$ \\
\hline
\end{tabular}

Valores expressos em média \pm desvio padrão; $C A=$ Circunferência abdominal; GJ = Glicemia de jejum; PAS = Pressão arterial sistólica; PAD = Pressão

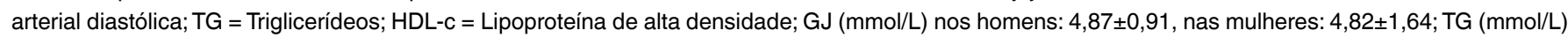
nos homens: $2,09 \pm 1,62$, nas mulheres: $1,58 \pm 0,84$; HDL-c (mmol/L) nos homens: $12,14 \pm 1,75$, nas mulheres: $12,99 \pm 2,19$.

Tabela 2. Comparação da prevalência total de Síndrome Metabólica em 675 agentes comunitários em uma cidade localizada no norte de Minas Gerais, segundo os critérios do NCEP-ATP III e IDF.

\begin{tabular}{lccc}
\hline & & IDF & Total \\
\hline NCEP-ATP III & Sim & Não & 227 \\
Nim & 221 & 6 & 448 \\
Total & 64 & 384 & 675 \\
\hline
\end{tabular}

NCEP-ATPIII = Third Report of the National Cholesterol Education Program; IDF = International Diabetes Federation; $p=0,000 ;$ índice Kappa = 0,781. 
Após análise da prevalência dos componentes da síndrome, notou-se que de acordo com o critério da IDF, após a CA (100\%), que é obrigatória para o diagnóstico, o componente mais prevalente no sexo masculino foi a PA $(95,3 \%)$ seguido pelo TG $(86 \%)$. Em relação ao sexo feminino, após a CA (100\%), a variável mais prevalente foi a PA $(90,9 \%)$, seguida pelo HDL-c $(61,6 \%)$ (Tabela 3$)$.

Para o critério do NCEP-ATP III, os componentes mais prevalentes foram a PA $(100 \%)$, TG $(90,9 \%)$ e CA $(69,7 \%)$ no sexo masculino. No sexo feminino, as três variáveis mais prevalentes foram CA $(94,3 \%), \mathrm{PA}(92,8 \%)$ e HDL-c (62,9\%) (Tabela 3).

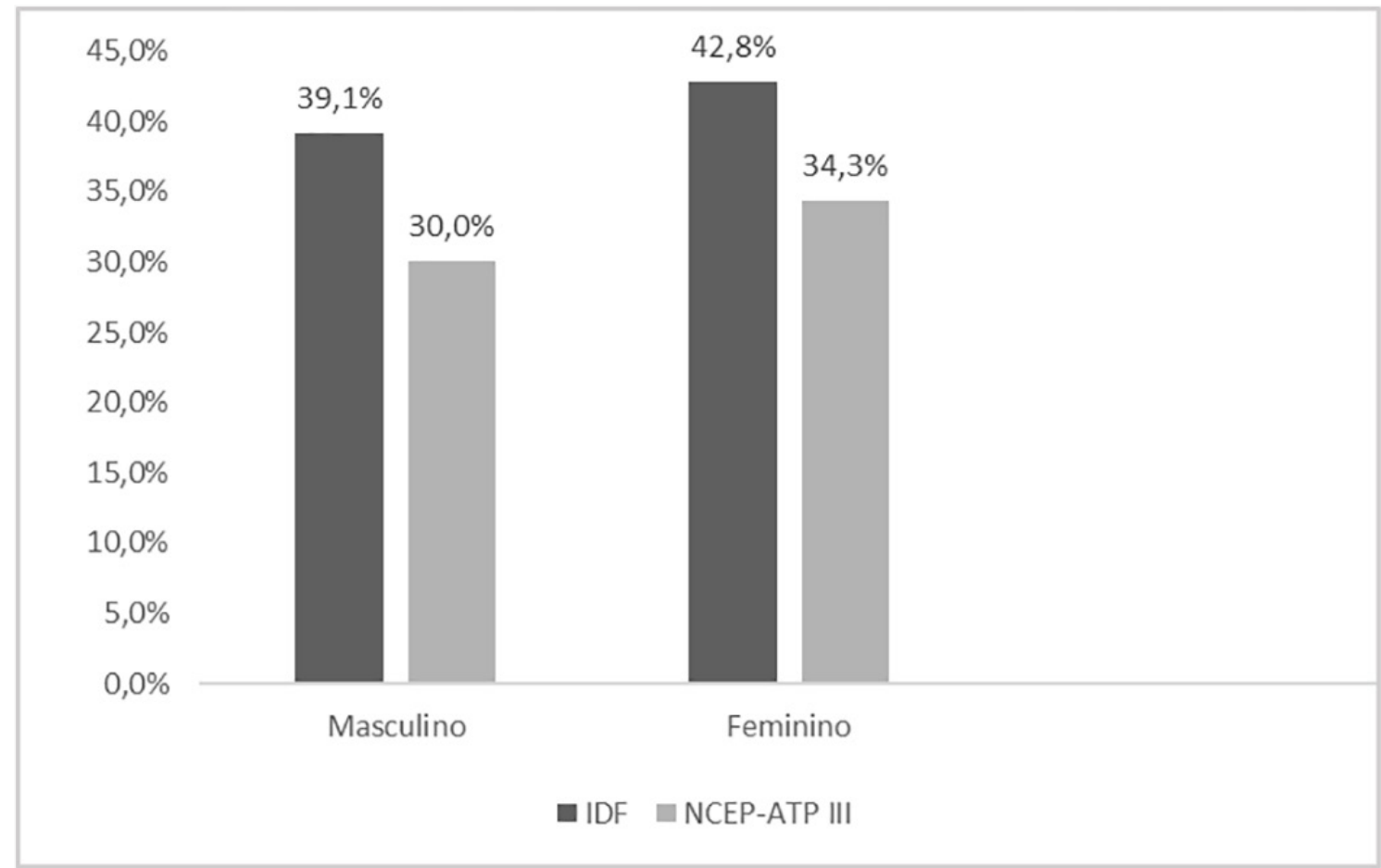

Figura 1. Prevalência da síndrome metabólica em relação ao sexo, de acordo com os critérios diagnósticos (homens = 110; mulheres = 565) $(n=675)$.

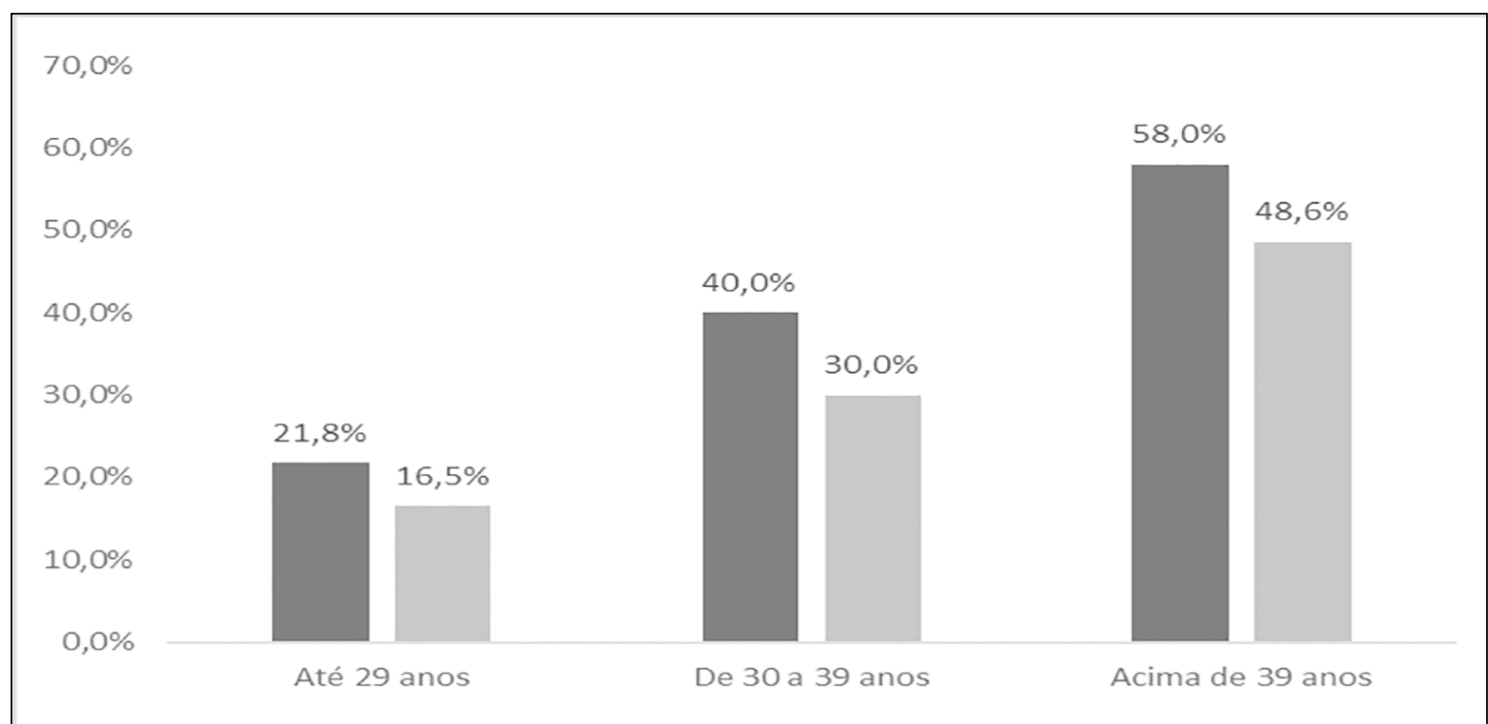

Figura 2. Prevalência da síndrome metabólica em relação à faixa etária, de acordo com os critérios diagnósticos (até 29 anos $=170 ;$ de 30 a 39 anos $=250 ;$ acima de 39 anos $=255)(n=675)$. 
Tabela 3. Distribuição da prevalência das variáveis da síndrome metabólica entre os agentes comunitários com SM em uma cidade localizada no Norte de Minas Gerais, pelo NCEP-ATPIII e IDF conforme gênero.

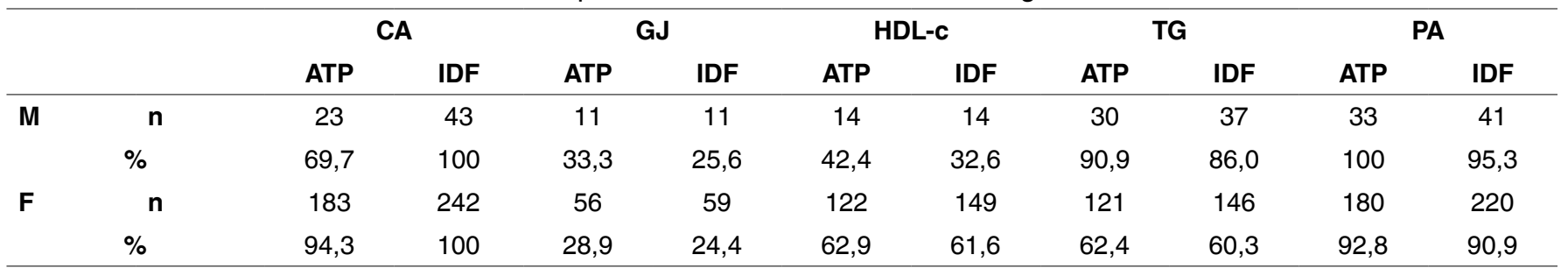

$\mathrm{M}=$ Masculino; $\mathrm{F}=$ Feminino $\mathrm{CA}=$ Circunferência abdominal; $\mathrm{GJ}=$ Glicemia de jejum; $\mathrm{HDL}=$ Lipoproteína de alta densidade; $\mathrm{TG}=$ Triglicerídeos; $\mathrm{PA}=\mathrm{Press}$ ão arterial; ATP $=$ Third Report of the National Cholesterol Education Program; IDF = International Diabetes Federation

\section{DISCUSSÃO}

A prevalência da SM foi consideravelmente alta pelos dois critérios diagnósticos utilizados, com maior prevalência na análise feita pela IDF em relação ao NCEP-ATP III. Análises realizadas em outros países demonstram diferentes estimativas de SM, como a pesquisa realizada por Wong-McClure et al. (2015), ${ }^{13}$ em cinco países da América Central, que apresenta uma prevalência geral de SM de 30,3\% somente pelo critério do NCEP-ATP III, sendo que nos cinco países avaliados a prevalência variou de $23 \%$ em Honduras a 35,1\% na Costa Rica. Por outro lado, Herningtyas e $\mathrm{Ng}(2019)^{14}$ demostraram prevalência de $21,66 \%$ na Indonésia, sendo maior ou igual a $20 \%$ em 11 das 20 províncias e em 19 dos 27 grupos étnicos analisados.

Resultados semelhantes a esse trabalho foram observados no estudo de Espósito et al. (2018), ${ }^{4}$ realizado com 500 homens na cidade de Natal, dos quais $60 \%$ foram diagnosticados com a doença, de acordo com a IDF, e 46,4\% de acordo com o NCEP-ATP III. Além disso, a concordância entre os critérios medida pela estatística Kappa $(k)$ foi de $(k=0,54)$ no estudo citado, o que evidencia boa compatibilidade entre eles, assim como no presente estudo.

Essa diferença se dá devido ao ponto de corte divergente para a obesidade central estabelecido pelos dois critérios utilizados - enquanto no NCEP-ATP III o ponto de corte é $88 \mathrm{~cm}$ para mulheres e $102 \mathrm{~cm}$ para os homens, na IDF é definido em $80 \mathrm{~cm}$ para mulheres e $90 \mathrm{~cm}$ para homens da América do Sul. A IDF propõe um valor específico para cada etnia analisada, o que permite abranger maior número de pessoas definidas como portadoras de SM ao adaptar os parâmetros à realidade das amostras estudadas. O NCEP-ATP III, ao apresentar valores de corte mais altos, desconsidera indivíduos que podem manifestar a síndrome em populações em que valores de corte racial específicos para a obesidade central são menores, como as asiáticas. ${ }^{1,5,6}$

Perante a comparação de porcentagem diagnóstica, levando em consideração o gênero masculino e feminino, notou-se insignificância estatística em ambos os critérios IDF $(p=0,467)$ e NCEP-ATP III $(p=0,378)$, devido à predominância feminina na população estudada $(83,7 \%)$, não sendo uma amostra estratificada por sexo. Estudos realizados por Almeida et al. (2016) ${ }^{15}$ e Barreto et al. (2018) ${ }^{16}$ também apresentam maior predominância do sexo feminino em populações de agentes comunitários de saúde, mostrando que este é um dado em diversas regiões pelo país.

Em sua pesquisa, Bortoletto et al. $(2016)^{17}$ evidenciaram a maior prevalência de SM pelo sexo feminino depois da sexta década de vida e diminuição da mesma para homens a partir da mesma idade. Esse aumento de prevalência nas mulheres foi correlacionado à diminuição do nível dos hormônios protetores e aumento do nível de colesterol total, gordura corporal e triglicerídeos no climatério. Já a diminuição 
de prevalência para os homens é atribuída à sobremortalidade masculina, ou seja, homens que atingem essa faixa etária apresentam menos exposição aos fatores de risco para a síndrome. ${ }^{18}$

Em relação à faixa etária, o presente estudo mostrou maior prevalência em ACS de acordo com o avançar da idade, resultado esse que foi estatisticamente significativo, coincidindo ao trabalho de Ramires et al. (2018). ${ }^{2}$ Essa característica da SM, geralmente, surge devido ao tempo de exposição aumentada a fatores de risco desta população. Isso aumenta o índice de SM nestes indivíduos, sendo necessárias medidas educativas de saúde para evitar complicações e aumento da morbimortalidade.

Alguns critérios diagnósticos para a SM, desenvolvidos ao longo do tempo, apresentam diferenças em seus componentes avaliados; porém, os analisados nesse estudo divergem apenas quanto aos pontos de corte. Esses componentes estão relacionados às complexas alterações fisiopatológicas no organismo e ao risco aumentado de DCV, sendo possível observar diferença na prevalência das variáveis de acordo com o critério utilizado e o sexo. ${ }^{10}$

A obesidade central é considerada o principal componente da SM, uma vez que contribui para ativação de cascatas inflamatórias, elevando a resistência insulínica e os efeitos das adipocinas, considerados fatores indutores de DCV. ${ }^{2,19}$ Nesta pesquisa, a CA está presente no grupo dos três componentes mais prevalentes pelo critério do NCEP-ATP III em ambos os sexos, sendo o mais encontrado no sexo feminino (94,3\%); em relação à IDF, devido à sua importância, é considerada componente obrigatório. O estudo de Salas et al. (2014), ${ }^{20}$ realizado no norte do México, também analisou a influência da CA, obtendo resultados concordantes, como a alta prevalência $(77,5 \%)$ e predominância no sexo feminino $(86,3 \%)$.

De acordo com o NCEP-ATP III, a PA apresentou a maior prevalência entre os componentes no sexo masculino e segundo mais prevalente no sexo feminino. Pelos critérios da IDF, excetuando-se a CA, foi o componente principal em ambos os sexos. Brajkovich et al. (2018) ${ }^{21}$ em uma análise realizada na Venezuela, demonstraram prevalência total da variável de 36,1\%, de acordo com o NCEP-ATP III, predominando também nos homens $(46,6 \%)$. O aumento da pressão arterial está diretamente relacionado ao envelhecimento, estando presente em $60 \%$ das pessoas acima de 65 anos, justificando o aparecimento de complicações, como a SM, principalmente em faixas etárias maiores. ${ }^{22}$

Observando-se os componentes relacionados à dislipidemia, nota-se uma relação significativa entre a hipertrigliceridemia e diminuição do HDL-c, presentes entre os três componentes mais prevalentes no sexo masculino e feminino, respectivamente, com a SM. Esses pacientes apresentam uma síntese aumentada de colesterol VLDL (lipoproteína de muito baixa densidade) e déficit no seu clearance, contribuindo para o aumento dos triglicérides, que se associa frequentemente a baixos níveis de HDL-c e altos níveis de partículas de colesterol LDL (lipoproteína de baixa densidade) pequenas e densas, predispondo à aterogênese e ao aumento da mortalidade. ${ }^{23}$

Embora a glicemia de jejum seja o componente menos prevalente nessa amostra, independente do sexo ou critério utilizado, duas análises na literatura demonstram a influência dessa variável. Após análise de amostras na Indonésia e Holanda, Sigit et al. (2020) ${ }^{24}$ apontam a GJ alterada como o segundo (51\%) e terceiro $(30,9 \%)$ componente mais prevalente na SM nessas populações, respectivamente. Esses resultados opõem-se ao presente estudo, demonstrando que há variações de acordo com as diferenças regionais da população analisada, porém, confirmam a relação entre as alterações do metabolismo da glicose e a presença da SM. 
Os ACS são expostos a inúmeras condições inadequadas de trabalho, como cargas biopsíquicas, que demonstram o impacto da profissão na saúde. A sobrecarga somática é feita de forma acumulativa, através do ritmo acelerado de trabalho, alimentação inadequada, redução da ingesta hídrica e tempo reduzido para realização de atividades físicas programadas, culminando no desenvolvimento de fatores de risco principalmente para condições crônicas, como a SM. É necessário relacionar essas cargas ao processo de adoecimento desses profissionais, a fim de reduzir a prevalência ou minimizar as complicações desses agravos. ${ }^{15}$

O presente estudo apresentou como limitação o fato de ter avaliado uma amostra restrita de agentes comunitários de uma única localidade, o que limita a expansão dos resultados para a população ACS em geral. A grande diferença do número de indivíduos do sexo feminino em relação ao masculino pode ter comprometido a análise comparativa entre os sexos, o que pode ser considerada uma limitação. Além disso, entre os critérios da IDF, incluem-se variáveis como presença de diabetes mellitus previamente diagnosticada, tratamento anti-hipertensivo e tratamento específico para dislipidemia. Tais dados não foram possíveis de serem colhidos em todos os agentes, sendo, assim, uma limitação. ${ }^{5}$

\section{CONCLUSÃO}

Neste estudo, foi encontrado porcentagem relevante de síndrome metabólica em agentes comunitários de saúde por pelo menos um dos critérios diagnósticos estudados. Percebeu-se uma compatibilidade entre os critérios diagnósticos da Internacional Diabetes Federation (IDF) e do National Cholesterol Education Program's Adult Treatment Panel III (NCEP-ATP III), que detectaram, respectivamente, $42,2 \%$ e $33,6 \%$ de pacientes com a síndrome. Uma amostra relevante que demonstra a disseminação da SM pela população estudada, sofrendo interferência estatisticamente significativa pelo aumento da faixa etária como demonstrado em outros estudos pelo Brasil.

Em relação às variáveis pesquisadas, pode-se afirmar que a circunferência abdominal, pressão arterial e os triglicerídeos se destacam como os fatores de risco mais prevalentes na síndrome em ambos os sexos e ambos os critérios diagnósticos avaliados. Esses dados facilitam o direcionamento intervencionista que se faz necessário para com a população de agentes comunitários de saúde que, além de serem exames fundamentais para se pesquisar, podem representar a triagem inicial para o diagnóstico precoce dos fatores de risco que podem culminar ao longo do tempo com o desenvolvimento da SM.

\section{Conflitos de interesse}

Os autores informam que não possuem conflitos de interesse.

\section{Financiamento}

Neste projeto houve o financiamento de bolsas de iniciação científica da Fundação de Amparo à Pesquisa do estado de Minas Gerais - FAPEMIG, Conselho Nacional de Desenvolvimento Científico e Tecnológico - CNPq e Centro Universitário FIPMoc (UNIFIPMoc). Não houve participação das instituições que contribuíram com as bolsas de iniciação científica no planejamento e condução da pesquisa, redação do artigo e decisão de publicar. 


\section{Contribuições na pesquisa}

Concepção e/ou delineamento do estudo: LDP, RRO, LGP, MONDA, ANOT, MFNS. Aquisição, análise ou interpretação dos dados: LDP, RRO, LGP, MONDA, ANOT, MFNS. Redação preliminar: LDP, RRO, LGP, MONDA, ANOT, MFNS. Revisão crítica da versão preliminar: LDP, RRO, LGP, MONDA, ANOT, MFNS. Todos os autores aprovaram a versão final e concordaram com prestar contas sobre todos os aspectos do trabalho. Sendo LDP, RRO, LGP, MONDA, ANOT, MFNS os acrônimos do nome dos autores.

\section{REFERÊNCIAS}

1. Saklayen MG. The global epidemic of the metabolic syndrome. Curr Hypertens Rep. 2018 Feb;20(2):12. DOI: https://doi.org/10.1007/s11906018-0812-z

2. Ramires EKNM, Menezes RCE, Longo-Silva G, Santos TG, Marinho PM, Silveira JAC. Prevalência e fatores associados com a síndrome metabólica na população adulta brasileira: pesquisa nacional de saúde - 2013. Arq Bras Cardiol. 2018 Mai;110(5):455-66. DOI: https://doi. org/10.5935/abc.20180072

3. Fortes MSR, Rosa SE, Coutinho W, Neves EB. Epidemiological study of metabolic syndrome in Brazilian soldiers. Arch Endocrinol Metab. 2019 Ago;63(4):345-50. DOI: https://doi.org/10.20945/2359-3997000000115

4. Espósito RC, Medeiros PJ, Silva FS, Oliveira AG, Aragão CFS, Rocha HAO, et al. Prevalência da síndrome metabólica segundo diferentes critérios na população masculina durante a Campanha Novembro Azul em Natal, RN, Nordeste do Brasil. Diabetes Metab Syndr Obes. 2018;11:401-8. DOI: https://doi.org/10.2147/DMSO.S168430

5. International Diabetes Federation (IDF). The IDF consensus worldwide definition of the metabolic syndrome [Internet]. Brussles: IDF; 2020; [acesso em 2020 Fev 17]. Disponível em: https://www.idf.org/e-library/consensus-statements/60-idfconsensus-worldwide-definitionof-themetabolicsyndrome.html

6. Expert Panel on Detection, Evaluation, and Treatment of High Blood Cholesterol in Adults. Executive summary of the third report of the National Cholesterol Eductation Program (NCEP) expert panel on detection, evaluation, and treatment of high blood cholesterol in adults (Adult Treatment Panel III). JAMA. 2001;285(19):2486-97. DOI: https://doi.org/10.1001/jama.285.19.2486

7. Siqueira FV, Reis DS, Souza RAL, Pinho S, Pinho L. Excesso de peso e fatores associados entre profissionais de saúde da Estratégia Saúde da Família. Cad Saúde Colet. 2019;27(2):138-45. DOI: https://doi.org/10.1590/1414-462x201900020167

8. Roth GA, Johnson C, Abajobir A, Abd-Allah F, Abera SF, Abyu G, et al. Global, regional, and national burden of cardiovascular diseases for 10 causes, 1999 to 2015. J Am Coll Cardiol. 2017 Jul;70(1):1-25. DOI: https://doi.org/10.1016/j.jacc.2017.04.052

9. Kubrusly M, Oliveira CMC, Simões PSF, Lima RDO, Galdino PNR, Souza PDAF, et al. Prevalência de síndrome metabólica diagnosticada pelos critérios NCEP-ATP III e IDF em pacientes em hemodiálise.J Bras Nefrol.2015 Mar;37(1):72-8. DOI: https://doi.org/10.5935/0101-2800.20150011

10. Malachias MVB, Souza WKSB, Plavnik FL, Rodrigues CIS, Brandão AA, Neves MFT, et al. $7^{\text {a }}$ Diretriz Brasileira de Hipertensão Arterial. Arq Bras Cardiol [Internet]. 2016 Set; [citado 2020-12-04]; 107(3 Supl 3):1-83. Disponível em: http://publicacoes.cardiol.br/2014/diretrizes/2016/05_ HIPERTENSAO_ARTERIAL.pdf

11. Landis JR, Koch GG. The measurement of observer agreement for categorical data. Biometrics. 1977 Mar;33(1):159-74. DOI: https://doi. org/10.2307/2529310

12. Ministério da Saúde (BR). Conselho Nacional de Saúde. Resolução no 510, de 07 de abril de 2016. Aprova diretriz e normas regulamentadoras de pesquisas envolvendo seres humanos [resolução na internet]. Diário Oficial da União, Brasília (DF), 24 maio 2016; [acesso em 2020 Fev 19]; Seção 1: 44-6. Disponível em: http://conselho.saude.gov.br/resolucoes/2016/Reso510.pdf

13. Wong-McClure RA, Gregg EW, Barceló A, Lee K, Abarca-Gómez L, Sanabria- López L, et al. Prevalence of metabolic syndrome in Central America: a cross-sectional population-based study. Rev Panam Salud Publica. 2015 Set;38(3):202-8.

14. Herningtyas $\mathrm{EH}, \mathrm{Ng} \mathrm{TS}$. Prevalence and distribution of metabolic syndrome and its components among provinces and ethnic groups in Indonesia. BMC Public Health. 2019 Apr;19:377. DOI: https://doi.org/10.1186/s12889-019-6711-7

15. Almeida MCDS, Baptista PCP, Silva A. Cargas de trabalho e processo de desgaste em agentes comunitários de saúde. Rev Esc Enferm USP. 2016 Fev;50(1):95-103. DOI: https://doi.org/10.1590/S0080-623420160000100013 
16. Barreto ICHC, Pessoa VM, Sousa MFA, Nuto SAS, Freitas RWJF, Ribeiro KG, et al. Complexidade e potencialidade do trabalho dos agentes comunitários de saúde no Brasil contemporâneo. Saúde Debate. 2018;42(spe1):114-29. DOI: https://doi.org/10.1590/0103-11042018s108

17. Bortoletto MSS, Souza RKT, Cabrera MAS, González AD. Síndrome metabólica, componentes e fatores associados em adultos de 40 anos ou mais de um município da Região Sul do Brasil. Cad Saúde Coletiva.2016 Mar;24(1):34-42. DOI:https://doi.org/10.1590/1414-462X201600010123

18. Dallazen F, Winkelmann ER, Berlezi EM. Risco cardiovascular avaliado pelo índice de conicidade em mulheres no climatério: análise comparativa entre os períodos pré e pós-menopausa. Sci Med. 2017 Dez;27(4):28268.

19. Know H, Kim D, Kim JS. Body fat distribution and the risk of incident metabolic syndrome: a longitudinal cohort study. Sci Rep. 2017 Set;7(1):10955. DOI: https://doi.org/10.1038/s41598-017-09723-y

20. Salas R, Bibiloni MDM, Ramos E, Vilarreal JZ, Pons A, Tur JA, et al. Metabolic syndrome prevalence among northern Mexican adult population. PLoS One. 2014;9(8):e105581.

21. Brajkovich I, González-Rivas JP, Ugel E, Rísquez A, Nieto-Matínez R. Prevalence of metabolic syndrome in three regions in Venezuela: The VEMSOLS Study. Int J Cardiovasc. 2018 Dez;31(6):603-9. DOI: https://doi.org/10.5935/2359-4802.20180065

22. Lima DBS, Moreira TMM, Borges JWP, Rodrigues MTP. Associação entre adesão ao tratamento e tipos de complicações cardiovasculares em pessoas com hipertensão arterial. Texto Contexto - Enferm. 2016;25(3):e0560015. DOI: https://doi.org/10.1590/0104-07072016000560015

23. Faludi AA, Izar MCO, Saraiva JFK, Chacra APM, Bianco HT, Afiune Neto A, et al. Atualização da diretriz brasileira de dislipidemias e prevenção da aterosclerose - 2017. Arq Bras Cardiol. 2017 Ago;109(1):1-76. DOI: https://doi.org/10.5935/abc.20170121

24. Sigit FS, Tahapary DL, Trompet S, Sartono E, Dijk KWV, Rosendaal FR, et al. The prevalence of metabolic syndrome and its association with body fat distribution in middle-aged individuals from Indonesia and the Netherlands: a cross-sectional analysis of two population-based studies. Diabetol Metab Syndr. 2020;12:2. DOI: https://doi.org/10.1186/s13098-019-0503-1 